\title{
Rendimiento en Prueba de Primado Semántico con Uso de Estímulos Palabra Condicionados
}

\author{
González-Gallo, Iván ${ }^{1(*)} ;$ Ariza, Sergio² \& Zedán-Salinas, Karina²
}

${ }^{1}$ Asociación Educar para el Desarrollo Humano. Laboratorio de Neurociencias y Educación. Buenos Aires, Argentina.

${ }^{2}$ Universidad Autónoma de Bucaramanga, Facultad de Psicología, Floridablanca, Colombia.

\section{RESUMEN}

La presente investigación se dirigió a corroborar si un estímulo/palabra, cuya función condicionada por medio de su emparejamiento con sonidos de alta intensidad de tipo demora corta y que elicita respuestas valoradas por los sujetos como indeseadas, ejerce control de la estimulación a modo de interferencia o facilitación de respuesta en una prueba de primado semántico cuyos estímulos target son palabras pertenecientes a las categorías deseado o indeseado. Se obtuvo un resultado significativo en la comparación de los grupos por medio de la prueba de Moses en los ensayos incongruentes observándose mayor cantidad de errores en los sujetos experimentales del grupo condicionado, sin embargo, no se encontraron diferencias significativas para el número de errores en ensayos congruentes y latencias en ninguno de los casos. Se explican en la discusión los posibles motivos por los que, a diferencia de los antecedentes de investigación, no se lograron resultados confirmatorios respecto a las latencias, abordando el significado y diferencias entre la no deseabilidad del estímulo condicionado en relación con la deseabilidad o ausencia de ésta de los estímulos target de la prueba, asimismo, los problemas de control dadas algunas limitaciones instrumentales.

Palabras clave: Conciencia; condicionamiento clásico; priming; prueba de interferencia

\section{Performance in Semantic Prime Test With Use of Conditioned Word Stimuli}

\section{ABSTRACT}

The present paper aimed to corroborate if a stimulus/word, whose conditioned function through its pairing with high-intensity sounds of short delay type and that elicits responses valued by the subjects as undesired, exerts control of the stimulation as interference or facilitation of response in a semantic primacy test whose target stimuli are words belonging to the desired or unwanted categories. A significant result was obtained in the comparison of the groups by means of the Moses extreme reactions test in incongruous trials, with a greater number of errors being observed in the experimental subjects of the conditioned group, however, no significant differences were found for the number of errors in trials congruent and latencies in none of the cases. The discussion explains the possible reasons why, unlike the research history, no confirmatory results were obtained regarding latencies, including the meaning and differences between the undesirability of the conditioned stimulus in relation to the desirability and undesirability of the Target stimuli of the test, likewise, control problems, given some instrumental limitations.

Keywords: Awareness; classical conditioning; priming; interference test. 


\section{INTRODUCCIÓN}

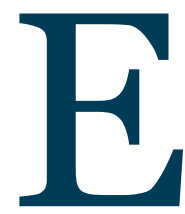

n la investigación psicológica del fenómeno nombrado como "Control Cognitivo", que comprende cualquier observación de secuencias de acciones inhibidas o suprimidas debido a cambios del entorno que señalizan secuencias antagónicas o simplemente distintas a las ya iniciadas, o como como la capacidad de direccionar y/o seleccionar esquemas de acción acordes a los cambios ambientales a modo de inhibición de respuestas emitidas hasta el momento o por cambios en los parámetros de las respuestas (Flores y Ostrosky-Shejet, 2012), se puede hallar consistentemente a lo largo sus publicaciones una metodología amparada en las pruebas de interferencia. Éstas pruebas consisten en la presentación un estímulo objetivo para el cual el individuo debe responder de manera adecuada según el criterio convenido por el experimentador, y dicha respuesta es dificultada por la presentación contigua o concurrente de otros estímulos interferentes. En estas pruebas los sujetos responden ante distintos ensayos que comportan arreglos de estímulos congruentes o incongruentes entre sí, en los que para el primer arreglo los estímulos se esperan faciliten la ejecución, mientras para el segundo, dificultarla o interferirla. Dentro de estas pruebas se encuentran las pruebas Stroop, Pruebas Go-no Go, la Tarea de Afecto Extrínseco de Simon, Tarea de Evitación de Acercamiento (Klauer, Stahl y Voss, 2012) entre otros. Por otro lado, estas pruebas pueden ser aplicadas utilizando estímulos tanto "conscientes" como "inconscientes", por ejemplo, usando una técnica de enmascaramiento para cubrir los estímulos interferentes cuya presentación, a su vez, se hace más corta; normalmente los normalmente con la asignación de cada letra para palabras positivas o negativas, funcionando como estímulos interferentes otra agrupación de palabras con valencia semántica que pueden tanto compartir como no compartir ejemplares con la batería de estímulos palabra objetivo. La presentación de los interferentes -o prime, es de tan solo una proporción de segundo y son enmascarados por un bloque que marca el lapso para que ocurra el estímulo palabra objetivo. La relevancia de este tipo de pruebas con presentaciones "fugaces" y enmascaradas de estímulos, cuya valencia semántica asociada puede ser congruente o incongruente con la asociada con los estímulos objetivo, hace parte de la inquietud sobre hasta qué punto la estimulación "inconsciente" puede alterar las actividades de los seres vivos. En especial cuando se parte del hecho de que en pruebas de interferencia con presentaciones explícitas, se observan efectos de facilitación o de interferencia (Reuss, Desender, Kiesel y Kunde, 2012).

En el presente trabajo, se realizó un cuasi-experimento dividido en dos fases. La primera, una fase de condicionamiento verbal similar a la metodología utilizada por Staats y Caso (1983) y por Olson y Fazio (2002), con la distinción con los segundos en que los emparejamientos se compusieron por estímulos palabra y sonidos de alta frecuencia, en vez de emparejamiento compuestos por únicamente palabras. Y la segunda, que consistió en la ocasión para que los sujetos respondieran ante distintos ensayos de interferencia o facilitación con el fin de identificar ¿Cuál es la incidencia de un estímulo condicionado recientemente por medio de un procedimiento de emparejamiento por demora corta en el control estimular cuando el individuo no pueda reportar su presencia?

\section{MARCO TEÓRICO}

En cercanía a una psicología operante, la conciencia es comprendida como el proceso en el que los individuos se percatan de lo que sucede en un entorno propio al organismo o diferenciado de éste, es decir, es un proceso por el que tanto las contingencias por las que se relacionan las variables ambientales que determinan la experiencia particular de un individuo, como las respuestas que son elicitadas o emitidas dentro de estas, por ejemplo, reconociéndose cuándo sucede una respuesta y qué tipo de respuesta, son advertidas. Esto implica hallar conductas cuyo antecedente control es otra conducta proveniente del mismo organismo o de las conductas de otros. De este modo, su estatus ontológico no es de entidad, sino un tipo más de movimiento de los organismos no ubicable en ningún lugar, tanto fuera como dentro de los individuos (Schlinger, 2008; Zilio 2011). Por su parte, Skinner refiere dos términos claves: conciencia verbal y no verbal. El primero consiste en responder discriminativamente de manera verbal al propio comportamiento y el segundo, se refiere a responder discriminativamente al propio comporta- 
miento (Skinner, 1974, citado por Zilio, 2011). Dicha proposición implica un aprendizaje de contingencias verbales según la comunidad lingüística en la que se encuentre la persona $y$, por otro lado, secuencias complejas de respuestas dentro de programas de reforzamiento no necesariamente verbal, como sucede en el término auto-conciencia (Pérez-Acosta, Rodríguez y Guzmán, 2002). Respecto a este último término, los procedimientos experimentales conocidos como encadenamiento muestran cómo una respuesta se torna estímulo discriminativo para otra, encajando en la definición de conciencia hasta el momento utilizada sin ser, eso sí, necesariamente dentro de un contexto socio-lingüístico. Sin más, esta aclaración final da a entender cómo coexisten la autoconciencia y la conciencia verbal sin solaparse puesto que "la conducta verbal fortalece la conciencia para un comportamiento autodescriptivo que es el corazón de un tipo diferente de conciencia" (Zilio, 2011, p. 11).

Por lo tanto, para el presente escrito los estímulos enmascarados presentados por el lapso descrito en el próximo apartado, son "inconscientes" en el sentido en que los sujetos no los advierten o, dicho de otra forma, no responden diferencialmente a éstos por medios verbales. Así mismo, dados los antecedentes de investigación en el uso de pruebas de interferencia con presentaciones "inconscientes", en los que se observaron efectos de facilitación o interferencia (Baños, Botella y Quero, 2000; La Heij, Schreuder y Van Der Heiden, 1985), en cierto sentido los sujetos son "conscientes" en la medida en que se comportan diferencialmente ante la presentación fugaz y enmascarada de los estímulos, aunque no puedan advertir de ello a través de un reporte de cualquier tipo. Los estímulos enmascarados se denominarán como "estímulos inadvertidos" o simplemente como estímulos enmascarados, aunque para remarcar el efecto que tiene su presentación, se utilizará el primer término.

Por otro lado, desde una posición más cercana a los programas de investigación de las ciencias cogniti

vas, Bechara, Damasio, Tranel, y Damasio (1997) señalan, desde un diseño experimental de línea base concurrente $A-B$ con un grupo de personas con lesiones prefrontales mediales, un posible proceso de consciencia como un conjunto de respuestas autónomas de señalización no consciente, que refleja el acceso a registros de experiencias individuales previas, aprendidas por condicionamiento y el estado emocional que le acompañan, los cuales progresivamente dan pie a sesgos de respuesta que a su vez, facilitan la toma de decisiones conscientes (Véase también Baños, Botella y Quero, 2000). Van Gaal, Lange y Cohen (2012) concuerdan en que "se ha demostrado que la información no consciente afecta varias funciones cognitivas perceptivas y de alto nivel y las áreas cerebrales asociadas, incluida la corteza prefrontal" (p.10). Lo cual refiere al Control Cognitivo, que se entiende como capacidad de direccionar y/o seleccionar esquemas de acción acordes a los cambios ambientales a modo de inhibición de respuestas emitidas hasta el momento o por cambios en los parámetros de las respuestas (Flores y Ostrosky-Shejet, 2012). Cabe señalar que, en dichos estudios, personas con lesiones prefrontales no se comportan en relación a los estímulos "inconscientes", es decir, no mostrando interferencia o facilitación en sus ejecuciones ante la presentación de estímulos que los individuos no puedan reportar ni tampoco correspondan a una representación perceptual visual. Por otro lado, en un análisis del estado del arte, se analizó conceptualmente la relación entre conciencia y funciones ejecutivas, encontrándose por ejemplo, que si la conciencia es definida como responder a las propias respuestas cognitivas o pensamientos, y hacerlo hallando o formando una identidad en ellas, toda área asociada la memoria episódica estaría materialmente relacionada con la conciencia, así mismo, se citan estudios sobre cómo el daño en estructuras dorso-laterales mediales de los prefrontales se relacionan con la organización del lenguaje, para conseguir objetivos específicos, y la reactividad emocional a estados emocionales ajenos, expresada en apatía e irritabilidad, los cuales son condición necesaria para algunos fenómenos de conciencia (Ardila, 2016). Todo lo anterior es importante considerando nuestro criterio de exclusión, en el que solo se aceptó la participación de personas aparentemente neurológicamente sanas y $\sin$ antecedentes reportados.

El papel que tiene el comportamiento consciente en el ajuste por el que pasa el resto del desempeño de los seres vivos con el ambiente es una temática estudiada tanto en producción disciplinar como extradisciplinar (Baldo, 2018), aquí se citan algunos antecedentes relevantes como la revisión del estado del 
arte realizada por Greenwall y Houwer en 2017 sobre el papel de la consciencia en el aprendizaje por condicionamiento clásico, como la línea de trabajo de Becker, Kleinböhl y Hölzl (2012) sobre la relación entre conciencia, aprendizaje operante y dolor crónico. En este último estudio se refiere un término relevante relacionado con la validez de las medidas de respuesta condicionada ( $R C$ ) en procedimientos de condicionamiento semántico, tal como señaló Staats en sus observaciones de que sus sujetos experimentales advertían que cierto estímulo solía seguir a otro (su línea de investigación en el tópico citada en Staats, 1996, citado en Becker, et. al. 2012), el cual para Becker refirió como "conciencia de contingencia". La conciencia de contingencia puede determinar la velocidad con la que una $\mathrm{RC}$ se adquiere a lo largo de los ensayos, como hacer posibles algunos aprendizajes (Greenwall y Houwer, 2017), tanto en condiciones operantes como respondientes; dicho término corresponde con la observación de enunciados de causa "si $x$, entonces $y$ " que explicita una relación de contingencia, es decir, relación de secuencias de eventos o relaciones causales entre eventos (Li y Poling, 2019) y que corresponde, en el caso de contingencias operantes, con el concepto de reglas (Skinner, 1981).

Respecto a la prueba de enmascaramiento semántico, se fijan relaciones entre las palabras de cada ensayo según antecedentes como proveniencia de un territorio con la misma lengua, condiciones psiquiátricas que pueden acompañarse de algún patrón de reactividad frente a ciertas clases de estímulo, como ocurre en los "sesgos", y valoraciones subjetivas pre test de algunos ejemplares de los estímulos antes de la contestación de los ensayos, entre otros, los cuales justificarán la nominación de los ensayos como incongruentes o congruentes. En relación a estos ensayos, se espera que para una tarea experimental de clasificación del significado de palabras estímulo a través de una tecla predefinida por el experimentador, en dos posibles respuestas o dos teclas, se observe una facilitación, es decir, una latencia inferior cuando los ensayos son congruentes, mientras que para los ensayos incongruentes se observa una interferencia, o una mayor latencia. Así mismo, dentro de estos diseños se pueden seleccionar palabras que para una comunidad tengan una valencia o significado por defecto, así como seleccionar aquellas valoradas como neutras en su dimensión "emocional" para utilizarlas en un procedimiento de condicionamiento y modificar su función; en algunos estudios (véase Olson y Fazio, 2001; Olson y Fazio, 2002) las palabras utilizadas como estímulos inadvertidos fueron tratadas en un emparejamiento para establecer una nueva función de estímulo, en la que la respuesta de los sujetos consistió en valoraciones como "negativo" o "positivo" referente a su valencia. Estos diseños de condicionamiento de palabras son similares a los implementados por Staats, aunque en algunos de sus estudios por condicionamiento del significado de las palabras los emparejamientos consistieron en palabras como estímulo condicional (EC) y choques eléctricos o sonidos de alta frecuencia como estímulo incondicional (EI) (Staats, Staats y Crawford, 1962, citados en Staats y Caso, 1983), con anterioridad se utilizaron emparejamientos de palabra-palabra (Staats, Staats y Biggs, 1958) y en otros casos los EC utilizados no tenían sentido semántico alguno, pues consistieron en secuencias de sílabas (Staats, Staats y Heard, 1961). Cabe preguntarse si según la historia interactiva del sujeto con estos estímulos implicados en el aprendizaje por condicionamiento clásico, tiene algún tipo de efecto en la ejecución dentro de estas pruebas de primado semántico. Debería haber alguna diferencia en usar palabras predefinidas por la comunidad como estímulo inadvertido a usar palabras recientemente condicionadas a través de su emparejamiento con otras palabras, de igual modo aquellas palabras emparejadas con estimulación aversiva proveniente de otros canales sensoriales (sonoro o táctil) y el uso de secuencias que en forma representan palabras, pero para la historia de aprendizaje de los sujetos no comporta un estímulo dotado de significado semántico. 


\section{METODOLOGÍA}

Se propone un diseño de corte cuasi-experimental transversal para el que se reclutan aleatoriamente 60 estudiantes del programa de psicología mayores de edad quienes previamente informados acepten formar parte de la prueba diligenciando el consentimiento. Los criterios de inclusión y exclusión referidos a los estudiantes de la Universidad Autónoma de Bucaramanga son: no reportar antecedentes de enfermedades graves ni en condiciones de discapacidades cognitivas o motrices; no pueden estar bajo el efecto de sustancias psicoactivas durante la aplicación de la prueba ni tener conocimiento previo de los códigos enmascarados. El sexo es indiferente y se requiere sean ciudadanos mayores de edad con conocimientos básicos en manejo computadores para participar en esta investigación cuantitativa.

La primera fase del experimento consiste en un programa de condicionamiento por emparejamiento de demora corta cuya contingencia es explícita, con motivo de establecer funciones condicionadas en la palabra 'Largo' (en el caso del grupo experimental) por lo que se exhorta a cada participante a cumplir una prueba de memoria en la que debe aprender la mayor cantidad de palabras posibles que aparezcan en pantalla. La sucesión de estímulos consistirá en los siguientes: dos códigos a condicionar, bloques blancos como estímulo interferente en identificación de contingencias, y variados ejemplares pertenecientes a categorías de estímulos comunes.

En la segunda fase, se realiza una prueba encubierta con la que se obtendrán medidas equivalentes al número de aciertos y latencia de respuesta. Para ello se les refiere una prueba de atención-reacción (The Subliminal Priming Task) en la que deben pulsar una de las dos claves de respuesta "positiva" o "negativa" (en el teclado) lo más rápido posible según las convenciones programadas para la valencia emocional de cada adjetivo por bloque (en total, 32 ejemplares tanto de valencia negativa como positiva establecidos convencionalmente). Actualmente la prueba ha sido digitalizada por la plataforma Millisecond Software que consiste en la asignación de cuatro categorías de estímulos a 2 claves de respuesta "agradable" y "desagradable". Adicionalmente, la prueba cuenta con un código subliminal que ha sido condicionado previamente con valencia negativa o como elicitador de respuestas de miedo, similar a lo descrito por Olson y Fazio (2002) y un código de enmascaramiento alfanumérico.

Finalmente se realiza una encuesta en la cual los sujetos deben evaluar la valencia emocional subjetiva elicitada por algunas palabras comunes que pudieran o no estar en los ensayos de condicionamiento iniciales. Esta medida se obtendrá para evaluar el condicionamiento inducido experimentalmente.

\section{RESULTADOS}

A continuación, se presentarán por medio de diagramas y tablas la descripción del desempeño de la muestra de estudio en la tarea de atención-reacción registrada en el software de la prueba The subliminal priming task. Para la medición se consideraron dos variables, siendo el número de aciertos y la latencia de respuesta según el tipo de emparejamiento (prime-adjetivo) que propone la prueba en cada grupo requerido en el diseño metodológico.

Para las respuestas emitidas durante los emparejamientos incongruentes, es decir, un prime de valencia emocional negativa y un adjetivo positivo, la distribución de datos es modal en 32 aciertos (siendo la puntuación máxima la que marca la tendencia), con un promedio de 31,30 aciertos en una muestra de 60 personas y una mediana $=31,50$. Por su parte, los datos tienden a dispersarse en (0,889 puntos). En cuanto a la forma, la distribución es leptocúrtica con una extensa cola hacia la izquierda característico de un amplio rango de datos por debajo de la media. Para observar el comportamiento general de la muestra en esta y demás variables contempladas en la prueba ver tabla 1 . Al separar los resultados, el grupo experimental $(n=27)$ cuenta con una amplitud similar a la del grupo control $(n=33)$, sin embargo, hay una mayor carga de datos en el límite inferior para el primer caso en comparación con el segundo grupo muestral. 
Tabla 1. Estadísticos descriptivos para variables de interés según tipo de respuesta (muestra general)

\begin{tabular}{|c|c|c|c|c|}
\hline & $\begin{array}{c}\mathrm{N}^{\circ} \\
\text { aciertos } \\
\text { incongrue } \\
\text { ntes }\end{array}$ & $\begin{array}{l}\text { Latencia } \\
\text { de } \\
\text { respuestas } \\
\text { incongrue } \\
\text { ntes }\end{array}$ & $\begin{array}{c}\mathrm{N}^{\circ} \\
\text { Aciertos } \\
\text { congruen } \\
\text { tes }\end{array}$ & $\begin{array}{c}\text { Latencia } \\
\text { de } \\
\text { respuesta } \\
\text { s } \\
\text { congruen } \\
\text { tes }\end{array}$ \\
\hline $\mathrm{N}$ & 60 & 60 & 60 & 60 \\
\hline Media & 31,30 & 739,98 & 30,25 & 773,73 \\
\hline $\begin{array}{l}\text { Median } \\
\mathrm{a}\end{array}$ & 31,50 & 697,00 & 30,50 & 736,50 \\
\hline Moda & 32 & $592^{\mathrm{a}}$ & $31^{\mathrm{a}}$ & $606^{\mathrm{a}}$ \\
\hline $\begin{array}{l}\text { Desv. } \\
\text { típ. }\end{array}$ & ,889 & 162,488 & 1,525 & 168,208 \\
\hline $\begin{array}{l}\text { Asimet } \\
\text { ría }\end{array}$ & $-1,538$ & 1,125 &,- 795 & 1,072 \\
\hline $\begin{array}{l}\text { Error } \\
\text { típ. de } \\
\text { asimetr } \\
\text { ía }\end{array}$ & ,309 & ,309 & ,309 & ,309 \\
\hline $\begin{array}{l}\text { Curtosi } \\
\mathrm{S}\end{array}$ & 2,743 & ,295 & ,372 &, 526 \\
\hline $\begin{array}{l}\text { Error } \\
\text { típ. de } \\
\text { curtosis }\end{array}$ & ,608 & ,608 & ,608 & ,608 \\
\hline
\end{tabular}

a. Existen varias modas. Se mostrará el menor de los valores.

Como se puede observar en la tabla 2, en la primera hipótesis se encontró una diferencia significativa con un alfa de $(0,05)$, es decir, las puntuaciones incongruentes extremas obtenidas en ambos grupos tienen un comportamiento independiente. En las pruebas para las hipótesis de 2 a 4, se encontraron valores que no representan un efecto plausible en la diferencia de los grupos por lo que dichas hipótesis no serán tratadas en este documento.
Tabla 2. Resumen de prueba de hipótesis no paramétrica

Hipótesis $\quad$ Sig. Decisión

$\begin{array}{lll}\text { El intervalo de } \mathrm{N}^{\circ} \text { aciertos } & 0,000^{1} & \begin{array}{l}\text { Rechazar la } \\ \text { incongruentes es el mismo } \\ \text { entre las categorías de }\end{array} \\ \text { nula }\end{array}$

Grupo

$\begin{array}{lll}\begin{array}{l}\text { El intervalo de } \mathrm{N}^{\circ} \text { aciertos } \\ \text { congruentes es el mismo } \\ \text { entre las categorías de }\end{array} & 0,082^{1} & \begin{array}{l}\text { Retener la } \\ \text { hipótesis } \\ \text { nula }\end{array}\end{array}$

Grupo

El intervalo de latencia de $\quad 1,000^{1} \quad$ Retener la respuestas incongruentes es hipótesis el mismo entre las nula categorías de Grupo

El intervalo de latencia de respuestas congruentes es el $\quad 0,563^{1} \quad$ Retener la mismo entre las categorías hipótesis de Grupo nula

Nota: en todos los casos se aplicó la Prueba de Mouses de reacción extrema de pruebas independientes.

Al aplicar la prueba de hipótesis se identificó que el rendimiento en la prueba según cantidad de aciertos es significativamente diferente cuando se trata de ensayos incongruentes. Dicha diferencia se observa en la acumulación de datos extremos en el grupo experimental que indica que en este grupo hubo menor cantidad de aciertos, el cual es un efecto atribuible a la función de respuesta establecida para el estímulo LARGO. Dicha función de respuesta pudo haber afectado en la ejecución de ensayos incongruentes en donde las respuestas asociadas a la palabra presentada de manera inadvertida, son incongruentes o antagónicas con las respuestas que están relacionadas con los estímulos consecuentes, que, según el diseño, tienen un significado emocional apetitivo. La significancia exacta con la que se rechaza la hipótesis nula es de $(0,000)$. 
Distribución de frecuencias para evaluación subjetiva

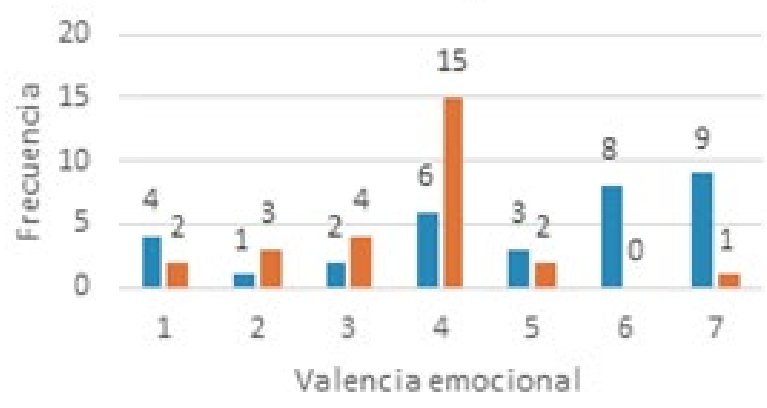

- Grupo experimental Grupo control

Figura 1. Diagrama de barras para prueba de hipótesis según valencia emocional subjetiva

Dentro del diseño metodológico se contempla una medida categórica obtenida por medio de una encuesta en la que los sujetos aprecian la valencia emocional de algunas palabras comunes, entre ellas la palabra condicionada "largo". La consistencia de la evaluación subjetiva sirve de soporte para retratar la fuerza de la asociación establecida durante los ensayos de condicionamiento semántico de primer orden, en este caso el grupo experimental establece una medición promedio de 4,91. El tercer y cuarto cuartil son ocupados por valoraciones de 7 puntos mientras que el grupo control obtienen un promedio de 3,59 . Los cuartiles tres y cuatro son ocupados por valores entre 4-7.

Al comparar las evaluaciones subjetivas según el grupo experimental al que pertenecen, se marca una tendencia a puntuar los valores más altos de la escala en los sujetos que pertenecen al grupo experimental. En el caso del grupo control el comportamiento de los datos para las categorías de evaluación subjetiva realizada por los sujetos en la última fase experimental tiene mayores indicaciones en puntuaciones medias y bajas de la escala. Teniendo en cuenta las características de la forma se aplica la prueba de Moses de reacción extrema para muestras independientes. Debido a la valencia emocional asignada por los sujetos en cada grupo asignado en la metodología se comprueba una diferencia significativa entre las puntuaciones para los grupos que conforman la muestra equivalente a $(0,012)$ contemplando a todos los sujetos participantes.

Partiendo de lo observado en la prueba de Moses que compara grupo $\mathrm{x}$ valencia emocional, se encontró una diferencia significativa en la evaluación subjetiva para la palabra LARGO, lo cual es coherente al logro de un establecimiento de función de respuesta por condicionamiento clásico o pavloviano. La diferencia entre valoración subjetiva del estímulo recae en que los sujetos del grupo condicionamiento, o experimental, tendían a responder a su valor máximo 7 , en el que se considera que los individuos perciben sus reacciones en relación a el estímulo visual, que comporta la palabra LARGO, como indeseados. Se considera como una medida presuntamente válida debido a que el procedimiento utilizado, al menos en términos de la naturaleza del estímulo neutro y el tipo de emparejamiento, son similares a los utilizados en diseños anteriores, los cuales señalan resultados significativos entre medidas de SCR y puntuación en escalas subjetivas (Staats, et al. 1962, citados en Staats y Caso, 1983), condiciones similares aunque sin medida objetiva (Staats, Staats y Biggs 1958) y diseños de emparejamiento similares más recientes (Dunsmoor, y LaBar, 2012; Dymond et al., 2011; y Declercq y De Houwer, 2009). De modo que, aunque el presente estudio no tuvo las medidas objetivas psicofisiológicas utilizadas en los antecedentes, como la conductancia eléctrica galvánica de miembros superiores, dada la consistencia entre medidas objetivas y de reporte en tales estudios, se consideró como válido utilizar como medida del condicionamiento las puntuaciones en la encuesta. Sin embargo, más adelante se harán algunos señalamientos a esta suposición.

Por otro lado, el uso de la prueba Moses para Datos Extremos arrojó únicamente distinciones en la comparación entre número de aciertos $\mathrm{x}$ grupo en ensayos incongruentes con una significatividad de $(=0,000)$. La diferencia en la cantidad de aciertos en dichos ensayos puede ser atribuida a la posible interferencia que comporta el inicio de una secuencia de respuestas antagónicas asociadas al prime, en relación a las respuestas asociadas al estímulo procedente o target. Sin embargo, esto es extraño en comparación con los resultados y fenómenos de 
primado que se han observado en otras investigaciones (Fuentes, Vivas y Humphreys, 1999) (Olson y Fazio, 2001; Olson y Fazio, 2006) (Becker, et al, 2012) donde hubo una clara interferencia o facilitación de la respuesta ante el estímulo target en términos de una disminución de la Latencia según el tipo de ensayo, es decir, congruente o incongruente; en estos estudios, a diferencia del presente, se utilizaron más de un estímulo inadvertido en la prueba de primado semántico, ya sea que probaran estímulos de contraria deseabilidad social posterior a un condicionamiento (Fazio y Olson, 2006), distintos estímulos pertenecientes a distintas categorías semánticas (Fuentes, et al. 1999) o que los estímulos-palabra usados como inadvertidos eran también presentados como estímulos target en posibles combinaciones (Becker, et al, 2012). Cabe resaltar que en la mayoría de las investigaciones, las palabras inadvertidas, o bien no fueron sujetas a una fase de condicionamiento sino seleccionadas por conocimiento de relaciones de categoría en la comunidad, o bien la fase de condicionamiento implicó emparejamientos de palabra-palabra, con o sin consciencia de contingencia, es decir, si el sujeto advertía que una palabra seguía a la otra; y cabe resaltar que la variable conciencia de contingencia, se considera como una condición necesaria para establecer una RC en unos casos, y en otros como una condición que lo impide, aunque el hecho de advertir una secuenciación pueda considerarse como otro tipo de RC (Lovibond y Shanks, 2011).

Por otro lado, Reuss, Desender, Kiesel y Kunde (2012) proponen dos puntos relevantes para la presente discusión. Primero, existen factores como el conflicto de frecuencia, que corresponde al incremento de la correcta ejecución a lo largo de los ensayos cuando la proporción de ensayos congruentes en relación a ensayos incongruentes es inferior, y caso contrario cuando la relación es inversa. Nuestro diseño consistió en la presentación de los ensayos de manera sucesiva y su tipo fue aleatorio, consistiendo en aproximado un $50 \%$ para cada tipo de ensayo. Segundo, a consideración de ellos la literatura podría estar permeada por un sesgo de publicación en el que las pruebas con resultados no confirmatorios del efecto de facilitación e interferencia serían menos selectos para publicar.
Finalmente, en relación a la medida subjetiva de condicionamiento clásico, se debe tener en cuenta las deficiencias en validez en la medida en que, probablemente, la categoría semántica en la que se incluyen las palabras utilizadas como objetivo, son de desfavorabilidad al igual que la usada como estímulo inadvertido, pero, divergiendo éstas en posibles clases más sutiles como de desfavorabilidad o favorabilidad social y desfavorabilidad de otro tipo. Esto es, el problema de que la categoría semántica de deseabilidad social que comporta la dicotomía "unpleasant/pleasant" en modo de adjetivos, no estén asociadas con una respuesta emocional similar a la respuesta de miedo probablemente elicitada por LARGO. Esto último es relevante debido a que ninguna palabra está relacionada especialmente con alguna respuesta emocional en particular, salvo, por ejemplo, NAUSEABUNDO que, a consideración de los autores del presente trabajo, puede que sí se acompañe de algunas respuestas de asco.

\section{CONCLUSIONES}

Es probable que sea conveniente replantear el diseño comparando las ejecuciones de grupos sometidos a distintas fases de condicionamiento, comparando la ejecución si la fase implicó emparejamiento de palabra-palabra, palabra-aversivo (sonoro o táctil) y palabras desconocidas, como las sílabas usadas por Staat, con otras palabras, así como establecer varias medidas de las posibles morfologías condicionadas que resulten de los emparejamientos.

Por otro lado, los efectos de facilitación o interferencia de la respuesta según los ensayos congruentes o incongruentes, respectivamente, identificada en otros estudios y revisada en el presente, podrían aplicarse en situaciones de marketing. Se podría revisar el efecto de la presentación de estímulos visuales de alta frecuencia (prime) en condiciones donde los sujetos deban elegir un producto electrodoméstico o de otra naturaleza, utilizando como estímulo inadvertido (prime) palabras que estén fuertemente ligadas con un elemento de interés pero no con otros (tomándolo de las asociaciones que se suelen proveer en las campañas publicitarias). Igualando las condiciones de elección al usar objetos de características de utilidad y precio similares, variando en parámetros secundarios de aspecto 
y marca, el uso de un estímulo-palabra inadvertido podría afectar la compra al "sesgar", por ejemplo, el tiempo en que el sujeto observa la ubicación de los productos de una misma marca, o alterando cualquier otra secuencia de comportamiento que pueda incrementar la tendencia de los compradores de adquirir un producto y no otro, o comprarle a una marca y no otra; también se podría simplificar la condición presentando todos los productos de elección en una sola página de catálogo informático.

En ese sentido, habiendo seleccionado la palabra fuertemente relacionada al producto o marca, se podría presentar en un punto de la pantalla que corresponda con la demostración explícita del producto o productos de una misma marca. Una medida de conducta que se puede hipotetizar como facilitadora de una decisión, podría ser los movimientos oculares, obtenida a través de los eye trackers. 


\section{REFERENCIAS}

Ardila, A. (2016). Is "self-consciousness" equivalent to "executive function"?. Psychology \& Neuroscience, 9(2), 215.

Baldo, M. (2018). Integración, teoría y método. En G. Gutiérrez (Ed.) (2018). Teorías en Psicología. Integración y el futuro de la disciplina. (34-101). Manual Moderno.

Baños, R., Botella, C. y Quero, S. (2000). Sesgos cognitivos en el trastorno de pánico: comparación entre el Stroop computerizado y con tarjetas. Psicothema. 12(2), 165-170. ISSN 0214 9915

Bechara, A., Damasio, H., Tranel, D., y Damasio, A. (1997). Deciding advantageously before knowing the advantageous strategy. Science, $275,1293-1295$

Becker, S., Kleinböhl, D., y Hölzl, R. (2012). Awareness is awareness is awareness? Decomposing different aspects of awareness and their role in operant learning of pain sensitivity. Consciousness and cognition, 21(3), 1073-1084.

Declercq, M., y De Houwer, J. (2009). Transfer of avoidance responding to a sensory preconditioned cue: Evidence for the role of SS and RS knowledge in avoidance learning. Learning and Motivation, 40(2), 197-208.

Dunsmoor, J. E., y LaBar, K. S. (2012). Brain activity associated with omission of an aversive event reveals the effects of fear learning and generalization. Neurobiology of learning and memory, 97(3), 301-312.

Dymond, S., Schlund, M. W., Roche, B., Whelan, R., Richards, J., y Davies, C. (2011). Inferred threat and safety: Symbolic generalization of human avoidance learning. Behaviour Research and Therapy, 49(10), 614-621.

Flores, L. J. C., y Ostrosky-Shejet, F. (2012). Desarrollo neuropsicológico de lóbulos frontales y funciones ejecutivas. Manual Moderno.

Fuentes, L. J., Vivas, A. B., y Humphreys, G. W. (1999). Inhibitory tagging of stimulus properties in inhibition of return: Effects on semantic priming and flanker interference. The Quarterly Journal of Experimental Psychology. Section A, 52(1), 149-164.
Gawronski, B., Deutsch, R. y Banse, R. (2012). Response Interference Tasks as Indirect Measures of Automatic Associations. En K. Klauer, A. Voss, y C. Stahl (Eds.) (2012). Cognitive methods in social psychology. (78-123). Guilford Press.

Greenwald, A. G., y De Houwer, J. (2017). Unconscious conditioning: Demonstration of existence and difference from conscious conditioning. Journal of Experimental Psychology: General, 146(12), 1705.

La Heij, W., Schreuder R. y Van Der Heiden A. (1985). Anticipación semántica e interferencia de tipo Stroop en tareas de denominación de palabras. Experimental Psychology: Human Perception and Performance. 11(1), 62-80.

Li, A. y Polling, A. (2019). Commit Heresy: Stop Using "Contingency". Behavior Analysis in Practice. 13, 226-231.

https://doi.org/10.1007/s40617-019-00354-5.

Lovibond, P.F., y Shanks, D.R. (2002). The role of awareness in Pavlovian conditioning: empirical evidence and theoretical implications. Journal of experimental psychology. Animal behavior processes, 28(1). 3-26.

Martens, U., Ansorge, U., y Kiefer, M. (2011). Controlling the unconscious: Attentional tasks sets modulate subliminal semantic and viso-motor priming differentially. Psychological Science, 22, 282-291.

Olson, M. y Fazio, R. (2001). Implicit attitude formation through classical conditioning. Psychological science. 12(5), 413-417.

Olson, M. y Fazio, R. (2002). Implicit acquisition and manifestation of classically conditioned attitudes. Social Cognition. 20(2). 89-103.

Olson, M. A., y Fazio, R. H. (2006). Reducing automatically activated racial prejudice through implicit evaluative conditioning. Personality and Social Psychology Bulletin. 32(4), 421-433.

Pérez-Acosta, A., Rodríguez, S. B., y Guzmán, J. N. (2002). Autodiscriminación condicional: la autoconsciencia desde un enfoque conductista. Revista Colombiana de Psicología, 11, 71-80. 
Reuss, H., Desender, K., Kiesel, A., y Kunde, W. (2012). Consciousness and cognitive control. Advances in Cognitive Psychology. 8(1), 9-18.

Schlinger, H. D. (2008). Consciousness is nothing but a word. Skeptic, 13(4), 58-63.

Reuss, H., Desender, K., Kiesel, A., y Kunde, W. (2012). Consciousness and cognitive control. Advances in Cognitive Psychology. 8(1), 9-18.

Schlinger, H. D. (2008). Consciousness is nothing but a word. Skeptic, 13(4), 58-63.

Skinner, B. F. (1981). Conducta verbal. Trillas. Staats, A. W., y Caso, M. (1983). Aprendizaje, lenguaje y cognición. Trillas.

Staats, A., Staats, C., y Biggs, D. (1958). Meaning of verbal stimuli changed by conditioning. The American journal of psychology, 71(2), 429-431.

Staats, A., Staats C. y Heard, W. (1961). Denotative meaning established by classical conditioning. Journal of experimental psychology. 61(4), 300-303..

Zilio, D. (2011). Consciência verbal, não-verbal e fenomênica: uma proposta de extensão conceitual no behaviorismo radical. Revista Brasileira de Terapia Comportamental e Cognitiva, 13(1), 4-19. 